\title{
Étude par chromatographie gazeuse inverse (CGI) des propriétés acido-basiques des matériaux textiles en verre
}

\author{
T. Hamieh, ${ }^{1,2}$, M. Gouider', M. Nardin ${ }^{1}$, J.-M. Senecot', J. Schultz', P. Christou ${ }^{3}$ \\ et P. Henrat ${ }^{3}$ \\ ${ }^{1}$ Institut de Chimie des Surfaces et Interfaces, ICSI-CNRS, UPR 9069, 15 rue Jean Starcky, \\ BP. 2488, 68057 Mulhouse cedex, France \\ ${ }^{2}$ Département de Chimie, Faculté des Sciences, Section 1, Université Libanaise, Hadeth, \\ Mont-Liban, Beyrouth, Liban \\ ${ }^{3}$ HEXCEL Fabrics, ZI Les Nappes, 38630 Les Avenières, France
}

\begin{abstract}
Some of our studies were devoted to characterise acid-base properties of some solid substrates like oxides : $\mathrm{MgO}, \mathrm{ZnO}, \mathrm{SiO}_{2}$ and $\mathrm{Al}_{2} \mathrm{O}_{3}$, carbon fibres and polymers as PMMA at various tacticities adsorbed on silica and on alumina by using inverse gas chromatography $(I G C)$ at infinite dilution, systems, that are known to interact strongly through acid-base interactions and ionic bonds. In this paper, we used $I G C$ technique to characterise three textile materials (in glass fibres), noted respectively $A, B$ and $C$. To do this, we used our model previously elaborated and giving the specific enthalpy of adsorption $\left(-\Delta H^{s p}\right.$ ) of a polar molecule adsorbed on a solid : $\left(-\Delta H^{S p}\right)=K_{A} \cdot D N+K D \cdot A N-K . A N \cdot D N$, were $D N$ and $A N$ are the electron donor and acceptor numbers of the molecule and $K_{A}$ and $K_{D}$ are the acidic and basic constants of the solid. The parameter $K$ reflects the amphoteric character of the solid. Values of $K_{A}, K_{D}$ and $K$ were obtained from this model by using Mathematica program, and allowed to give the following conclusions :

- Material $A$ has an acidic character and it is more acidic than the two other materials.

- Material $B$ has an amphoteric character with a basic tendency.

- Fibre $C$ is the more basic material relatively to the others, the ratio between its basic constant to acidic constant being close to 3 .
\end{abstract}

\section{INTRODUCTION}

Nous assistons depuis plusieurs dizaines d'années à une évolution constante et à une utilisation croissante des matériaux composites. Ces matériaux ont l'avantage d'associer de hautes performances mécaniques à leur légèreté, permettant ainsi de remplacer, pour de nombreux usages, les matériaux ordinaires tels que les métaux. Leur application se diversifie et entre dans tous les domaines de la vie quotidienne ainsi que dans d'autres plus spécifiques (aéronautique, automobile, militaire, santé...).

Le bon emploi des composites est fortement dépendant de l'adhésion établie entre leurs deux constituants (le renfort fibreux et la matrice). Ainsi l'étude des propriétés superficielles de ces constituants et des phénomènes d'adhésion à leur interface est primordiale, afin de prévoir la résistance des composites et essayer d'accroître leurs performances. 
Les méthodes de caractérisation des surfaces sont en constante évolution et deviennent de plus en plus performantes et précises. Parmi ces méthodes, la chromatographie gazeuse inverse à dilution infinie constitue une bonne méthode de caractérisation permettant l'accès aux paramètres thermodynamiques de la surface des solides divisés sous forme de poudre ou de fibre. Cette méthode constitue le moyen d'étude, que nous avons choisie, afin de déterminer les caractéristiques de surface de tissus en fibres de verre non-traités et traités silanes.

\section{PRESENTATION DES TISSUS ETUDIES}

L'utilisation des composites renforcés par les fibres de verre est très variée. On peut les trouver dans tous les domaines allant des applications très spécifiques à des applications grand public. Parmi ces applications, on peut citer : travaux publics et bâtiment, électricité et électronique, sports et loisirs, transports, secteurs militaires... Les fibres de verre sont souvent employées avec des résines thermodurcissables notamment les résines époxy. L'une des applications de ce type de composites concerne les plaques de support électronique [1] (circuits imprimés...) constituées, en général, d'un empilement de plusieurs couches de tissus de verre E préimprégné de résine époxy bromée. Les tissus que nous avons étudiés sont, en effet, utilisés pour cette application. Nous avons analysé trois tissus (Hexcel) en verre $\mathrm{E}$, le premier est un tissu nu désensimé thermiquement, les deux autres sont constitués par ce même tissu mais traités silanes. Ils sont désignés respectivement par : tissu $A, B$ et $C$.

\section{TECHNIQUE UTILISEE ET METHODES}

\section{1 la chromatographie gazeuse inverse}

La chromatographie en phase gazeuse $[2,3]$ est une méthode de séparation des gaz et des composés susceptibles d'être vaporisés à une température appropriée. Parmi les différents types de chromatographie en phase gazeuse, nous nous intéressons à la chromatographie Gaz/Solide d'adsorption. C'est une technique couramment utilisée pour analyser des mélanges complexes de produits organiques (Figure 1). Deux phases sont mises en jeu, une phase stationnaire solide sous forme de poudre ou de fibre, contenue dans une colonne en acier inoxydable de quelques millimètres de diamètre et d'une longueur qui peut atteindre quelques mètres appelé colonne, traversée en permanence par la phase mobile gazeuse (souvent de l'hélium ou de l'azote) appelé aussi gaz vecteur. Contrairement aux méthodes analytiques classiques qui s'intéressent à la phase gazeuse injectée sur une phase solide bien définie, notre but est d'étudier les propriétés de surface de la phase solide par l'injection de sondes (molécules gazeuses) bien connues. De ce fait, la méthode a été nommée chromatographie gazeuse inverse ( $C G I)$.

Les sondes sont, dans la grande majorité des cas, des molécules organiques. Dès leur injection à l'entrée de l'appareil, les sondes se diluent dans le gaz vecteur qui les entraîne à travers la colonne. Les interactions moléculaires entre les solutés et la phase stationnaire freinent ces molécules, qui se déplacent ainsi moins vite que le gaz vecteur. En outre, celles-ci se déplacent à des vitesses différentes, selon l'intensité des interactions. Il en résulte qu'elles émergent de la colonne à tour de rôle sous la forme de pics d'autant plus élargis que leur temps de transit est plus long (Figure 1).

La CGI à dilution infinie consiste à injecter de très faibles quantités de sondes (à la limite de la détection) l'une après l'autre. L'adsorption des molécules gazeuses se fait d'une manière réversible à la surface du support, et on peut considérer le mélange gazeux comme un gaz parfait, donc toute interaction entre les molécules sondes sont nulles qu'elles soient adsorbées ou désorbées [4-6].

L'appareil [7] que nous avons utilisé est un chromatographe en phase gazeuse classique muni d'un dispositif d'enregistrement permettant le traçage des pics de sortie (Figure 1). Les deux 
extrémités de la colonne sont raccordées l'une à l'injecteur, l'autre au détecteur tous deux thermostatés.

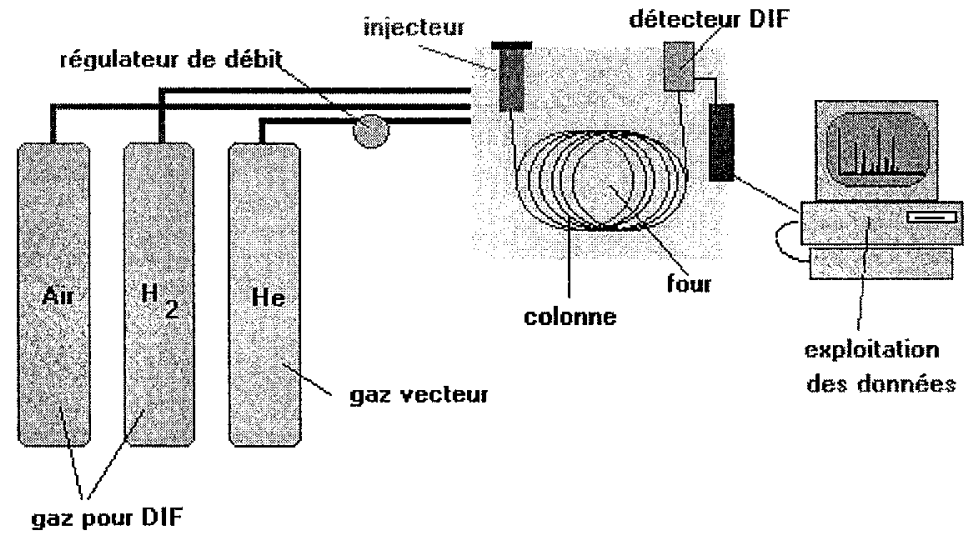

Figure 1. Schéma d'un chromatographe muni d'un détecteur à ionisation de flamme $(D I F)$

Pour avoir la possibilité de travailler à des températures différentes, la colonne est placée dans un four dont la commande est autonome. La mesure du débit du gaz vecteur est assurée par un débitmètre à film de savon qui est lié à la sortie de la colonne. Ce type de débitmètre donne un résultat très précis et peut servir à l'étalonnage de tous les autres débitmètres [7]. En général, le débit choisi lors d'une étude en CGI est compris entre 20 et $40 \mathrm{~cm}^{3} / \mathrm{mn}[4-6,8-10]$.

\subsection{Grandeurs de rétention}

La grandeur expérimentale en $C G I$ est le temps de rétention $t_{R}$. C'est le temps qui sépare l'instant de l'injection et celui de l'apparition du sommet du pic (Figure 2) dans le cas de pics symétriques. Chaque sonde a son propre temps de rétention qui dépend de ses interactions avec la phase solide. Cependant, un gaz non retenu, comme le méthane, suit le gaz vecteur à travers la colonne et émerge après un certain temps appelé temps mort $t_{o}$ (Figure 2).

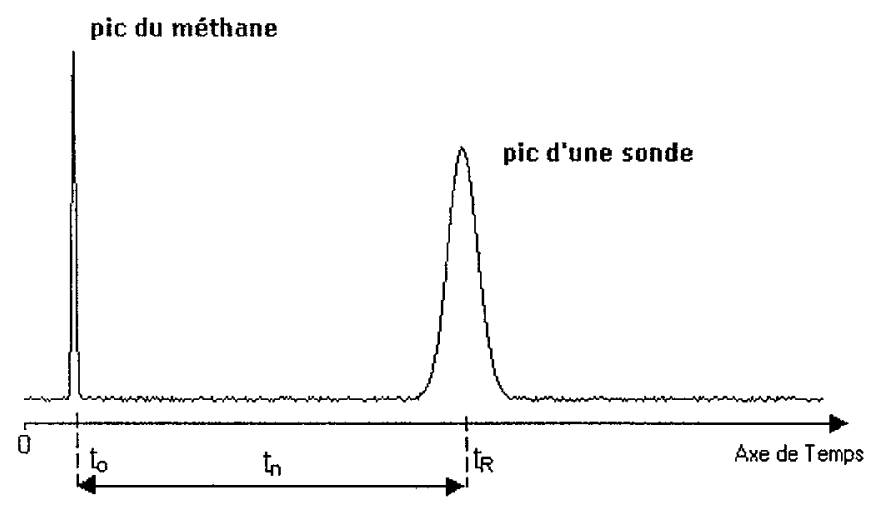

Figure 2. Chromatogramme classique 
Ainsi, définit-on le temps net de rétention $t_{n}=t_{R}-t_{o}$ pour chaque sonde qui traduit uniquement son temps de séjour sur la surface du support chromatographique.

Le volume net de rétention $V_{n}$, qui sert à déterminer les grandeurs thermodynamiques, correspond au volume de gaz vecteur qui traverse la colonne depuis l'introduction de la sonde jusqu'à la sortie du maximum diminué du volume mort $V_{o}$ de la colonne. Il est lié au temps de rétention par la relation suivante :

$$
V_{n}=D_{c}\left(t_{R}-t_{o}\right)
$$

Avec $D_{c}$, le débit corrigé donné par la relation suivante :

$$
D_{c}=j D_{m i j}\left(1+\frac{P_{H_{2} O}}{P_{a}}\right) \frac{T_{c} \eta\left(T_{a}\right)}{T_{a} \eta\left(T_{c}\right)}
$$

$D_{m}$ est le débit mesuré à l'aide du débitmètre, $T_{a}$ et $T_{c}$ sont respectivement la température ambiante au moment de mesure du débit et la température de travail de la colonne, $P_{a}$ et $P_{\mathrm{H}_{2} \mathrm{O}}$ sont respectivement la pression atmosphérique lors de l'étalonnage et la pression de vapeur saturante de l'eau à $T_{a}$, et $\eta\left(T_{c}\right)$ et $\eta\left(T_{c}\right)$ sont respectivement la viscosité du gaz vecteur à la température ambiante et de travail. Le coefficient $j$ de James-Martin tient compte de la compressibilité du gaz dans la colonne sous l'action de la perte de charge $\Delta P, P_{a t m}$ étant la pression atmosphérique le jour du travail :

$$
j=\frac{3}{2} \frac{\left(1+\Delta P / P_{a t m}\right)^{2}-1}{\left(1+\Delta P / P_{a t m}\right)^{3}-1}
$$

\subsection{Précautions à prendre}

La validité des résultats dépend essentiellement du bon déroulement de l'expérimentation, et le respect de quelques règles notamment :

$\varpi$ Le chromatogramme doit être linéaire et reproductible, c'est-à-dire que la forme et la position des pics ne varie pas avec la quantité de sonde injectée. Dans ce cas, il est possible de considérer le soluté comme un gaz parfait, donc les interactions entre les molécules sondes sont négligeables.

co Le chromatogramme doit être le plus idéal possible, c'est-à-dire qu'on doit obtenir des pics fins et symétriques, mais la non-idéalité inévitable provoque l'élargissement des pics et parfois leur dissymétrie causée par des phénomènes de diffusion. Dans ce cas, il faudrait corriger à l'aide du moment du premier ordre.

* Le volume net de rétention pour chaque sonde doit rester constant si on change le débit du gaz vecteur. En d'autres termes, si le débit augmente alors le temps de rétention d'une sonde donnée doit diminuer d'une manière à avoir le même volume net de rétention.

\subsection{Calcul des interactions spécifiques par la méthode SAINT-FLOUR et PAPIRER [12, 13]}

Cette méthode permet le calcul des interactions spécifiques $\Delta \mathrm{G}^{\mathrm{sp}}$ entre chacune des sondes polaires et la surface du solide. Ce paramètre est déterminé par la représentation du logarithme du volume de rétention des sondes gazeuses en fonction du logarithme de leurs tensions de vapeur saturante. Sur cette représentation, on place tout d'abord les points concernant les alcanes, dans ce cas on obtient une droite. La figure 3 représente un exemple appliqué à l'un des tissus analysés dans notre étude. 
Bien que cette méthode soit empirique, elle est justifiée dans la mesure où l'étude se veut avant tout témoin de l'évolution des propriétés de surface des tissus.

La deuxième étape consiste à porter sur le même graphique les points relatifs aux sondes polaires. Pour chacune d'entre elles, l'écart entre le point expérimental et la droite des alcanes donne une appréciation de ses interactions spécifiques avec la surface du solide (Figure 3).

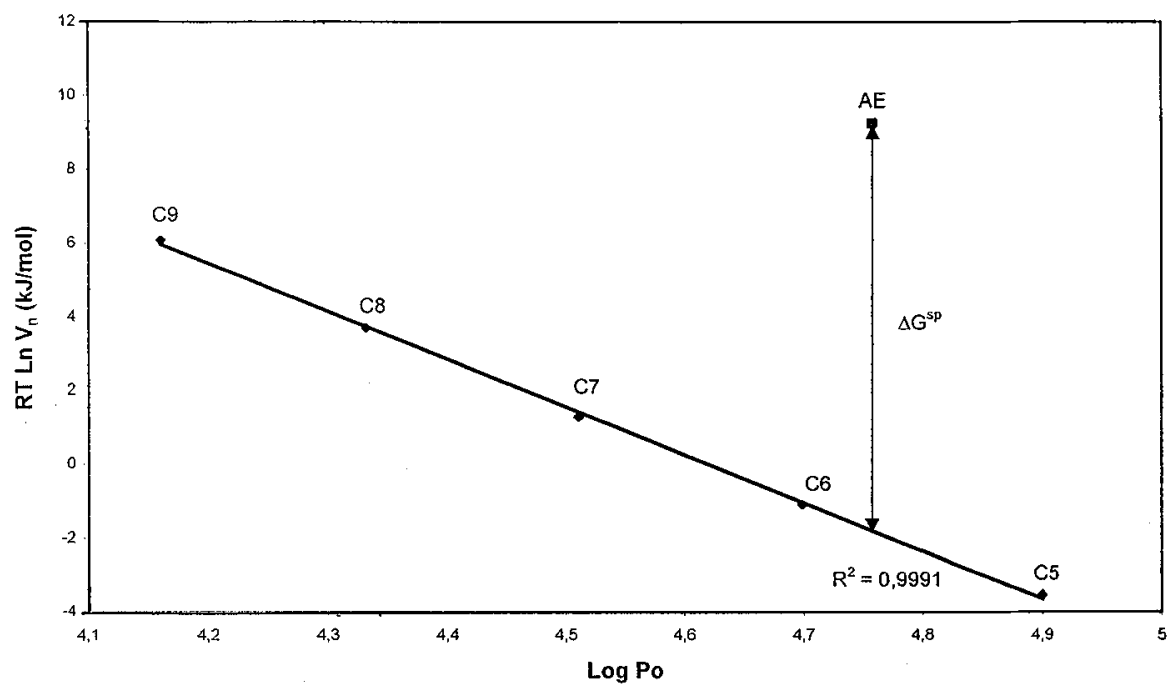

Figure 3. Variation du logarithme du volume net de rétention des n-alcanes en fonction du logarithme de leur tension de vapeur saturante pour un tissu non traité à $40,3^{\circ} \mathrm{C}$.

\section{RESULTATS EXPERIMENTAUX}

\subsection{Evaluation du caractère acido-basiques d'un solide $[9,10]$ : Modèles théoriques}

L'approche la plus utilisée concernant le caractère acido-basique des sondes utilisées est celle de Gutmann qui attribue à chacune d'entre elles un nombre donneur ND (J/mol) et un nombre accepteur NA (sans dimension) d'électrons, qui sont des paramètres semi-empiriques [11]. Cette approche tient compte du caractère amphotère des sondes, mais on lui reproche la non homogénéité des deux nombres. Par analogie avec Gutmann [11], Papirer et coll.[12-14] ont proposé de caractériser le solide par deux constantes, acide $\mathrm{K}_{\mathrm{A}}$ et basique $\mathrm{K}_{\mathrm{D}}$. L'interaction acide-base de ce solide avec la sonde se traduira par une enthalpie spécifique :

$$
-\Delta \mathrm{H}^{\mathrm{sp}}=\mathrm{K}_{\mathrm{A}} \cdot \mathrm{ND}+\mathrm{K}_{\mathrm{D}} \cdot \mathrm{NA}
$$

$\Delta \mathrm{H}^{\mathrm{Sp}}$ peut être déterminée en traçant $\Delta \mathrm{G}^{\mathrm{sp}}$ déterminée par CGI en fonction de la température $T$ $\left(\Delta \mathrm{G}^{\mathrm{sp}}=\Delta \mathrm{H}^{\mathrm{sp}}-\mathrm{T} . \Delta \mathrm{S}\right)$, en supposant que $\Delta \mathrm{H}^{\mathrm{sp}}$ ne varie pas avec la température dans le domaine exploré. Récemment, Hamieh et al. [5,6] ont normalisé les unités de ND et NA en les rendant tous les deux adimensionnels; ils proposent alors une correction pour le nombre donneur selon $\mathrm{ND}^{\prime}=2,5 \mathrm{ND}$. D'autre part, Riddle \& Fowkes ont proposé une correction du nombre accepteur NA' des sondes [15] pour enlever la contribution des forces dispersives (type Van Der Waals). Le 
nombre accepteurs NA' n'étant jamais nul, on peut alors représenter, pour toutes les sondes, $\frac{-\Delta H^{s p}}{N A^{\prime}}$ en fonction de $\frac{N D^{\prime}}{N A^{\prime}}$ qui doit être une droite en raison de la relation (4). Les constantes $K_{A}$ et $K_{D}$ sont alors estimées respectivement par la pente de la droite obtenue et son ordonnée à l'origine. Par ailleurs, Hamieh et al.[6] ont corrigé l'approche de Papirer (relation (4)) par l'ajout d'une constante $\mathrm{K}\left(\mathrm{K}_{\mathrm{A}}, \mathrm{K}_{\mathrm{D}}\right)$ qui traduit le couplage entre le caractère acide et basique du solide étudié, tenant ainsi compte de son caractère amphotère. En effet, les interactions acide-base entre une surface solide et une molécule amphotère ne se font pas totalement entre cette dernière et tous les sites superficiels du solide. Il y a donc une certaine partie de ces sites qui n'échangera pas des interactions avec la molécule sonde. La relation proposée s'écrit :

$$
-\Delta H^{s p}=K_{A} \cdot N D^{\prime}+K_{D} \cdot N A^{\prime}-K \cdot N A^{\prime} \cdot N D^{\prime}
$$

En divisant cette relation par $\mathrm{NA}^{\prime}$, on aura :

$$
-\frac{\Delta H^{s p}}{N A^{\prime}}=K_{A} \frac{N D^{\prime}}{N A^{\prime}}+K_{D}-K . N D^{\prime}
$$

Cette équation s'écrit symboliquement :

$$
\begin{gathered}
\mathrm{X}_{1}=\mathrm{K}_{\mathrm{D}}+\mathrm{K}_{\mathrm{A}} \mathrm{X}_{2}-\mathrm{K}_{3} \\
\mathrm{X}_{1}=\frac{-\Delta \mathrm{H}^{\mathrm{sp}}}{\mathrm{NA^{3 }}}, \quad \mathrm{X}_{2}=\frac{\mathrm{ND}^{\prime}}{\mathrm{NA}^{3}}, \mathrm{X}_{3}=\mathrm{ND}^{\prime} \text { et } \mathrm{K}=\mathrm{K}\left(\mathrm{K}_{\mathrm{A}}, \mathrm{K}_{\mathrm{D}}\right)
\end{gathered}
$$

où $\mathrm{X}_{1}, \mathrm{X}_{2}$ et $\mathrm{X}_{3}$ sont connus pour chaque molécule polaire utilisée, alors que $\mathrm{K}_{\mathrm{D}}, \mathrm{K}_{\mathrm{A}}$ et $\mathrm{K}$ sont les inconnues du problème.

Si l'on utilise $\mathrm{N}$ molécules sondes, la relation (7) permet alors d'écrire les équations suivantes :

$$
\begin{gathered}
\sum_{=1}^{N}\left(\mathrm{X}_{1}\right)_{\mathrm{i}}=\mathrm{K}_{\mathrm{D}} \mathrm{N}+\mathrm{K}_{\mathrm{A}} \sum_{\mathrm{i}=1}^{\mathrm{N}}\left(\mathrm{X}_{2}\right)_{\mathrm{i}}-\mathrm{K} \sum_{\mathrm{i}=1}^{\mathrm{N}}\left(\mathrm{X}_{3}\right)_{\mathrm{i}} \\
\sum_{=1}^{N}\left(\mathrm{X}_{1} \mathrm{X}_{2}\right)_{\mathrm{i}}=\mathrm{K}_{\mathrm{D}} \sum_{\mathrm{i}=1}^{N}\left(\mathrm{X}_{2}\right)_{\mathrm{i}}+\mathrm{K}_{\mathrm{A}} \sum_{\mathrm{i}=1}^{\mathrm{N}}\left(\mathrm{X}_{2}\right)_{\mathrm{i}}^{2}-\mathrm{K} \sum_{\mathrm{i}=1}^{N}\left(\mathrm{X}_{2} \mathrm{X}_{3}\right)_{\mathrm{i}} \\
\sum_{=1}^{N}\left(\mathrm{X}_{1} \mathrm{X}_{3}\right)_{\mathrm{i}}=\mathrm{K}_{\mathrm{D}} \sum_{\mathrm{i}=1}^{\mathrm{N}}\left(\mathrm{X}_{3}\right)_{\mathrm{i}}+\mathrm{K}_{\mathrm{A}} \sum_{\mathrm{i}=1}^{\mathrm{N}}\left(\mathrm{X}_{2} \mathrm{X}_{3}\right)_{\mathrm{i}}-\mathrm{K} \sum_{\mathrm{i}=1}^{N}\left(\mathrm{X}_{3}\right)_{\mathrm{i}}^{2}
\end{gathered}
$$

On a ainsi un système linéaire de trois équations (8-10) à trois inconnues qui sont $\mathrm{K}_{\mathrm{D}}, \mathrm{K}_{\mathrm{A}}$ et $\mathrm{K}$. La matrice représentant cette application linéaire est symétrique, donc il existe une solution unique pour $N \geq 3$.

\subsection{Résultats concernant les interactions spécifiques}

Devant la difficulté et l'incertitude de l'évaluation des aires d'adsorption des molécules sondes, nous avons choisi la méthode de St-Flour \& Papirer pour étudier les propriétés acido-basiques des différents tissus. L'étude de $R T \operatorname{Ln} V_{n}$ en fonction de $L n P_{o}$ pour les différents conduit à évaluer les interactions spécifiques des différentes sondes avec ces tissus $(A, B$ et $C)$. Les résultats obtenus montrent, d'une part, que la surface des trois tissus interagissent avec les trois types de sondes (acide, basique et amphotère), donc on peut dire, en première approximation, que ces surfaces sont 
amphotères. D'autre part, on peut noter que les interactions spécifiques du tissu $A$ avec les sondes basiques et amphotères sont nettement plus importantes que celles des autres tissus. En revanche, c'est le tissu $C$ qui présente les interactions spécifiques les plus élevées avec les sondes acides, suivi du $B$. De plus, on remarque que les tissus $A$ et le $B$ interagissent à peu près de la même manière avec l'acétone $\left(N D^{\prime}=42,5\right.$ et $\left.N A^{\prime}=8,7\right)$ et l'acétate d'éthyle $\left(N D^{\prime}=42,75\right.$ et $\left.N A^{\prime}=5,3\right)$ et que les tissus $B$ et $C$ interagissent à peu près de la même manière avec l'éther $\left(N D^{\prime}=48\right.$ et $\left.N A^{\prime}=4,9\right)$ et le THF $\left(N D^{\prime}=50\right.$ et $\left.N A^{\prime}=1,9\right)$. On peut alors prévoir que les tissus $A$ et $\mathrm{B}$ auront leurs coefficients $K$ de couplage acido-basique équivalents et que les tissus $B$ et $C$ auront leur constante d'acidité $K_{A}$ équivalente. La détermination des constantes $K_{A}, K_{D}$ et $K$, détaillée au paragraphe suivant, nous donnera une idée plus claire sur ces points.

\subsection{Etude de l'acido-basicité des tissus}

Nous avons pu remonter aux constantes d'acido-basicité en appliquant les deux méthodes (classique de Papirer et nouvelle de Hamieh) explicitées au chapitre précédent. Les résultats obtenus montrent que la variation des enthalpies libres spécifiques $\Delta G^{s p}$ des différentes sondes sont à peu près constantes. La contribution du terme entropique dans l'expression de $\Delta G^{s p}$ étant négligeable, nous n'en avons pas tenu compte dans la suite de notre travail.

\subsubsection{Méthode classique}

La méthode classique de Papirer et coll. nous a permis de déterminer les contantes $K_{A}$ d'acidité et $K_{D}$ de basicité pour les trois tissus. Le tableau I présente les constantes acide $K_{A}$ et basique $K_{D}$ pour les différents tissus :

Tableau I : Les constantes d'acido-basicité des différents tissus déterminées par l'ancienne méthode.

\begin{tabular}{|c|c|c|}
\hline Tissu & $K_{A}(\mathrm{~J} / \mathrm{mol})$ & $K_{D}(\mathrm{~J} / \mathrm{mol})$ \\
\hline$A$ & 174,7 & 164,8 \\
\hline$B$ & 107,6 & 205,3 \\
\hline$C$ & 78,4 & 265,2 \\
\hline
\end{tabular}

on constate que le tissu $A$ a un caractère amphotère avec une légère prédominance acide, le tissu $B$ est basique et le tissu $C$ est fortement basique. Ce résultat est en cohérence avec le comportement de chaque tissu avec les molécules d'eau à savoir: le premier est très hydrophile, le second est hydrophobe et le troisième est très hydrophobe. Ainsi le traitement des tissus permet de protéger ces derniers contre l'humidité, ceci est très important, puisque le tissu va être employé comme renfort pour les plaques électroniques.

\subsubsection{Nouvelle méthode}

Le calcul des constantes $K_{A}, K_{D}$ et $K$ des différents tissus a été réalisé à l'aide d'un logiciel de calculs mathématiques. Les résultats sont présentés dans le tableau II. 
Tableau II : Les constantes d'acido-basicité des différents tissus déterminés par la nouvelle méthode

\begin{tabular}{|c|c|c|c|c|}
\hline Tissu & $K_{A}$ & $K_{D}$ & $K$ & $\begin{array}{c}\text { Coefficient de } \\
\text { corrélation }\end{array}$ \\
\hline$A$ & 160,5 & 83,7 & 7,1 & 0,99 \\
\hline$B$ & 94,5 & 129,7 & 6,6 & 0,988 \\
\hline$C$ & 70,3 & 219,7 & 4,1 & 0,987 \\
\hline
\end{tabular}

Les valeurs obtenues, pour $K_{A}$ et $K_{D}$, par cette méthode sont peu différentes de celles obtenues par l'ancienne approche, à l'exception des $K_{D}$ des tissus $A$ et $B$. Pour ces derniers, le coefficient $K$ est élevé. L'examen de ces résultats nous conduit aux conclusions suivantes :

Le tissu $A$ a un caractère acide et il est nettement supérieur à celui des deux tissus traités. Sa constante d'acidité est deux fois plus importante que sa constante de basicité.

G. Le tissu $B$ a un caractère amphotère avec une légère tendance basique.

Le tissu $C$ a un caractère fortement basique avec une constante de basicité trois fois plus importante que sa constante d'acidité.

Les coefficients de couplage $K$ des tissus $A$ et $B$ sont à peu près égaux. On remarque aussi que les constantes d'acidité $K_{A}$ des tissus $B$ et $C$ sont relativement proches. Ces résultats confirment nos prévisions du paragraphe précédent sur les interactions spécifiques.

D'autres études $[16,17]$ ont été effectuées sur ces fibres en vue de déterminer la montée ou progression capillaire (verticale et horizontale) des liquides à l'intérieur des fibres de textile en verre.

\section{CONCLUSION}

Dans le cadre de ce travail, nous avons pu comparer les propriétés superficielles des tissus en verre E nu et traité silanes. L'étude a été effectué par chromatographie gazeuse inverse à dilution infinie. A partir de l'injection de très faibles quantités de sondes apolaires et polaires nous sommes parvenus à apprécier, pour les trois tissus, le caractère acido-basique au sens de donneur-accepteur d'électrons en leur attribuant des constantes d'acidité $K_{A}$ et de basicité $K_{D}$. Concernant l'acidobasicité des surfaces, nous avons pu remarquer que le tissu nu a un caractère acide et que les traitements, augmentent considérablement sa basicité surtout dans le cas du traitement $C$. La CGI, bien qu'elle soit une méthode qualitative, donne de bons résultats qui sont cohérents avec les résultats de la littérature et parfaitement adaptés à une comparaison entre différents solides. Mais, il est très important de maîtriser tous les paramètres mis en jeu, notamment, l'éventuel changement de 
l'aire des molécules des sondes, ainsi que la dilatation thermique des solides étudiés, surtout dans le domaine des hautes températures. Ces paramètres sont jusqu'à maintenant négligés ou mal pris en compte.

\section{Références Bibliographiques}

[1] Busch R. and Philibert T., Composites, 7 (1997) 48-53

[2] Conder J.R. and Young C.L., Physical measurements by gas chromatography. J. Wiley and Sons, New York, 1979.

[3] Riddle F.L. and Fowkes F.M., J. Am. Chem. Soc, 112(9) (1990) 3259-3264.

[4] Hamieh T. et Schultz J., J. Chim. Phys., 93 (1996) 1292-1331.

[5] Hamieh T., C. R. Acad. Sci., Série II b, 325 (1997) 353-362

[6] Hamieh T., Nardin M., Lescouët M., Haïdara H. and Schultz J., Colloïdes and Surfaces, 125 (1997) 155-161

[7] Tranchant J., Manuel pratique de chromatographie en phase gazeuse, Masson \& Cie, Paris, 1964.

[8] Belgacem M. N. and Gandini A., Surfactant Science Series, 80 (1991) 41-124

[9] Smidsrod O., Guillet J.E., Macromolecules, 2(3) (1969) 272-277.

[10] Schultz J., Lavielle L., Martin C., J. Chim. Phys. 87 (2) (1987) 231.

[11] Gutmann V., The donor-acceptor approach to molecular interactions, Plenum Press, 1978.

[12] Saint-Flour C. and Papirer E., Ind. Eng. Chem. Prod. Res. Dev. 21 (2) (1982) 337-341.

[13] Papirer E. and Saint-Flour C., J. Colloid. Interf. Sci., 91 (1) (1983) 69-75.

[14] Nardin M. and Papirer E., J. Colloid. Interface Sci., 137 (2) (1990) 534-545.

[15] Riddle F.L. and Fowkes F.M., J. A. Chem. Soc., 112 (9) (1990) 3259-3264.

[16] SENECOT J-M., Nardin M., REITER G., SCHULTZ J., CHRISTOU P. and HENRAT P. "Capillary rise in glass fabrics", EURAD 2000, LYON, 2000.

[17] SENECOT J-M., Nardin M., REITER G., SCHULTZ J., CHRISTOU P. and HENRAT P. "The capillary progression in glass fabrics: 1) Geometrical parameters", Publication en cours. 